Z. Yin, P. Nau, W. Meier, Responses of combustor surface temperature to flame shape transitions in a turbulent bi-stable swirl flame, Exp. Thermal Fluid Sci. 82 (2017) 50-57.

The original publication is available at www.elsevier.com

http://dx.doi.org/10.1016/j.expthermflusci.2016.11.004 


\title{
Responses of combustor surface temperature to flame shape transitions in a turbulent bi-stable swirl flame
}

\author{
Zhiyao Yin ${ }^{\mathrm{a}, *}$, Patrick Nau ${ }^{\mathrm{a}}$, Wolfgang Meier ${ }^{\mathrm{a}}$ \\ ${ }^{a}$ Institute of Combustion Technology, German Aerospace Center (DLR), Pfaffenwaldring 38-40, 70569, Stuttgart, \\ Germany
}

\begin{abstract}
Turbulent swirl flames are known to exhibit intermittent transitions between V-and M-shapes at certain operating conditions. A diagnostic apparatus based on a commercial highspeed laser system is developed in this work to investigate the influence of such transient phenomenon on the surface temperature of an atmospheric-pressure gas turbine model combustor. The transition of flame shapes is monitored by planar OH laser-induced fluorescence (OH PLIF) at 1-kHz repetition rate. Simultaneously, the same laser beam is used to perform 1-D surface thermometry on the base plate and the bluff body of the combustor, which are coated with $\mathrm{Mg}_{4} \mathrm{FGeO}_{6}$ : $\mathrm{Mn}$, a commonly used thermographic phosphor (TP). Temperature is inferred from the decay of phosphorescence recorded by a highspeed CMOS camera operated at $70 \mathrm{kHz}$. During transitions between attached $(\mathrm{V}-)$ and lifted (M-) flames, pronounced temperature variation as much as $100 \mathrm{~K}$ is observed on the tip of the bluff body. In the spectral domain, temperature measured during the M-flame period exhibits a peak at $475 \mathrm{~Hz}$, in good agreement with the measured frequency of the precessing vortex core (PVC) at the same operating condition. This peak does not appear during the V-flame period when PVC is suppressed. The uncertainty of the measurements and the sensitivity of the technique are discussed in detail.
\end{abstract}

Keywords: Surface Thermometry, Thermographic Phosphor, Highspeed Laser Diagnostics, Turbulent Swirl Flames

\section{Introduction}

Confined turbulent flames are commonly encountered in practical combustion systems such as gas turbines and IC engines. Studies of these systems have traditionally treated the confinement (combustor surfaces) as isothermal or adiabatic, while focusing primarily on its role in combustion aerodynamics and acoustics. In recent years, increasing attention has been drawn to heat loss to the confinement, and its influences on combustion chemistry and flame stabilization [1] as well as on flame flashback [2]. Consideration of heat loss to surfaces have proven critical in improving large eddy simulations (LES) for different combustor configurations [1, 3]. Such considerations may also be needed for understanding and predicting unsteady phenomenons such as periodic fluctuations and transient behaviors in certain combustion systems.

This work concentrates on such a phenomenon that is encountered in swirl stabilized flames, namely the intermittent transitions between attached (V-shape) and lifted (M-shaped) flames at socalled bi-stable operating conditions [4, 5]. The transition from V-to M-flame occurs as a sequence

\footnotetext{
${ }^{*}$ Corresponding author

Email address: zhiyao.yin@dlr.de (Zhiyao Yin)
} 
of local extinction near the flame root, formation of a helical precessing vortex core (PVC) [6] and an eventual total lift-off. The opposite happens when PVC is suppressed and flame becomes attached again. PVC has been well characterized for turbulent swirl flames and is recognized as an essential player in combustion stabilization [7]. As the formation of PVC is highly sensitive to the local density (temperature) field [4], knowledge of temperature on flame holders (e.g., bluff body) is important for gaining a full picture of the stochastic nature of these events.

The aim of this work is to develop a diagnostic apparatus to resolve, temporally and spatially, the effect of flame shape transitions on the surface temperature of a swirl-stabilized combustor. With the rapid development of surface thermometry using thermographic phosphors (TP) [8, 9] and highspeed laser/acquisition systems, various approaches have been recently demonstrated to achieve spatially[10-15] and temporally-resolved [16] surface temperature measurements in different combustion environments. These approaches have conventionally employed harmonic outputs from YAG lasers and are generally decoupled from other laser-based techniques. For the current purpose, however, direct correlation between transient combustion events and responses of surface temperature is required. In this work, planar laser-induced fluorescence of $\mathrm{OH}$ radical (OH PLIF) is used to monitor flame shape at $1 \mathrm{kHz}$. Simultaneously, surface thermometry is carried out by using the same UV laser to excite the TP coated on the base plate and the bluff body of the combustor. The focus is placed on identifying the location which experiences the largest temperature variations during flame transitions. The uncertainty of the measurements and the sensitivity of the technique are also addressed in detail.

\section{Experimental setup}

\subsection{Combustor and operating condition}

The atmospheric-pressure gas turbine model combustor used in this work is based on an industrial design by Turbomeca and has been the subject of extensive experimental [17-19] and numerical [20-22] investigations. The combustor, as illustrated in Fig.1 1 , was operated at a perfectly premixed mode [18] in this work. Methane and air mixture at an equivalence ratio of $\phi=0.7$ and a thermal loading of $20 \mathrm{~kW}$ was first delivered into the cylindrical plenum $(\varnothing=78 \mathrm{~mm})$ of the combustor. It then went through a swirl generator with 12 radial vanes before entering into the combustion chamber via a converging nozzle $(\varnothing=27.85 \mathrm{~mm})$ with a central conical bluff body $(\varnothing=29 \mathrm{~mm}$ at the bottom and 34-mm tall). The combustion chamber featured a square cross-section of $85 \times 85 \mathrm{~mm}^{2}$, 114-mm tall and was capped such that combustion products exited through a conical converging section into an exhaust duct $(\varnothing=40 \mathrm{~mm})$. The side walls of the chamber were made of quartz, providing access to optical diagnostics. A sonic nozzle was installed upstream of the plenum to isolate the combustor acoustics from that of the gas supply lines. For the operating condition, the Reynolds number at the exit of the nozzle based on the average cold flow exit velocity of $14.4 \mathrm{~m} / \mathrm{s}$ and the nozzle diameter, is about 28,000. The swirl number, derived from the velocity measurements at 1.5 $\mathrm{mm}$ above the exit is approximately 0.6 [17].

At the thermal loading of $20 \mathrm{~kW}$, flame was observed to transition from being attached at the bluff body (V-shape) to being completely lifted off (M-shape) as equivalence ratio was reduced from stoichiometric to $\phi=0.65$ [4]. At the target operating condition of $\phi=0.7$, flame alternated randomly between $\mathrm{V}$ - and $\mathrm{M}$-shape (bi-stable). Figure 2 shows averaging $\mathrm{OH}$ chemiluminescence images illustrating the $\mathrm{V}$ - and M-flames taken at this condition. $\mathrm{OH}$ chemiluminescence signal was acquired using an intensified CMOS camera (LaVision HSS5 with HS-IRO, active sensor size $512 \times 512$ pixels, $40 \mu$ s gate) coupled with an achromatic UV lens ( $f=64 \mathrm{~mm}, \mathrm{f} / 2.0$, Bernhard Halle Nachfl.) and a bandpass filter $(300-325 \mathrm{~nm})$. The camera was operated at $1 \mathrm{kHz}$ in order to resolve 
Figure 1:

(a) Schematic of the gas turbine model combustor

(b) Arrangement of the diagnostic system

(c) Single-shot raw image of phosphorescence at room temperature overlaid on top of a detailed sketch of the burner surface (not to scale)

(a)

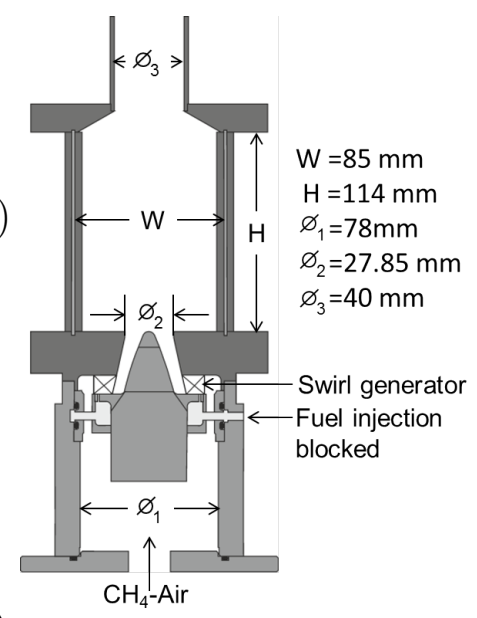

(b)

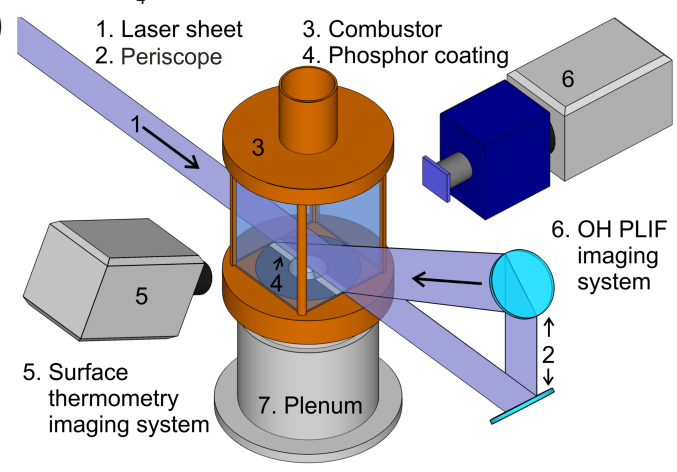

(c)

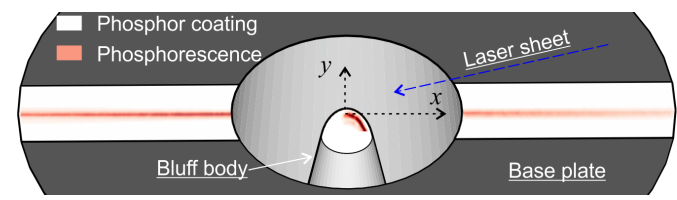

the transitions between the two flame shapes. The two images in Fig 2 were obtained by averaging frames collected during either of the flame periods. As can be seen, while flame is directly attached at the bluff body during the V-shape period, it becomes lifted during the M-shape period.

\subsection{Diagnostic arrangement}

The layout of the combined OH PLIF and 1-D surface thermometry is shown schematically in Fig.17. A commercial highspeed laser system consisted of a Sirah Cobra-Stretch HRR dye laser pumped by the second harmonic output from an Edgewave IS-811E diode-pumped solid state Nd:YLF laser. The dye laser was frequency doubled and tuned to $283.2 \mathrm{~nm}(1 \mathrm{kHz}, 50 \mu \mathrm{J} /$ pulse $)$ to coincide with the Q1(7) transition in the OH A-X $(1,0)$ band. For OH PLIF, the UV beam was expanded into a laser sheet with two cylindrical lenses $\left(f_{1}=-50 \mathrm{~mm}\right.$ and $\left.f_{2}=250 \mathrm{~mm}\right)$, which

Figure 2:

Average $\mathrm{OH}$ chemiluminescence taken during the $\mathrm{V}$ - and M-flame periods
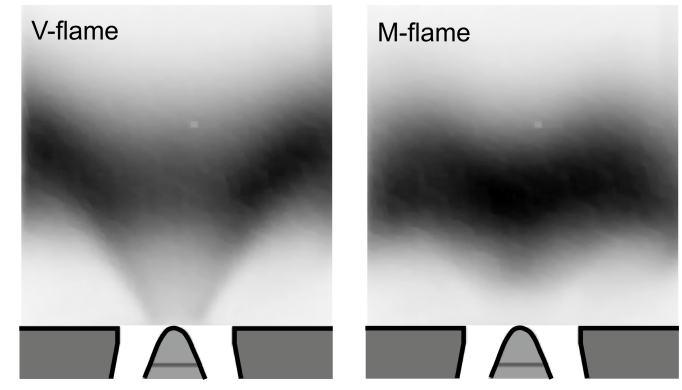
Figure 3:

Timing diagram of the UV laser pulse and the camera gate for capturing phosphorescence

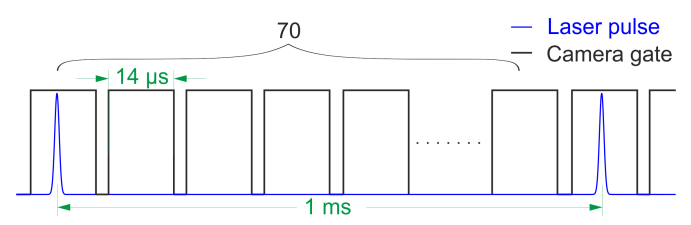

was then softly focused waist-wise with an $f_{3}=1000 \mathrm{~mm}$ cylindrical lens and aligned across the geometric center of the combustion chamber. After passing through the chamber, the same laser sheet was redirected via a periscope to excite the TP coating on the surface of the combustor for thermometry. The forward and backward laser sheets were overlapped spatially such that the obtained surface temperature could be correlated to the $\mathrm{OH}$ signal distribution. The laser sheet was adjusted to slowly expand vertically, from about $50 \mathrm{~mm}$ tall for OH PLIF to about $80 \mathrm{~mm}$ tall for surface thermometry.

OH PLIF signal was collected using the same camera system described above for OH chemiluminescence imaging, but with a 100-ns intensifier gate. Prior to each set of measurements, average PLIF signal from Acetone vapor was obtained as a correction for laser sheet profile. Phosphorescence (centered at about $656 \mathrm{~nm}$ ) was collected by a CMOS camera (LaVision HSS8, active sensor size $960 \times 40$ pixels) equipped with a Canon lens $(f=85 \mathrm{~mm}, \mathrm{f} / 1.4)$. The camera was suspended at about $60^{\circ}$ relative to the burner surface. No additional interference filter was used since the lens is not transmissive to the wavelength of the UV beam. The coordinate system used in this work is defined in Fig.1 b, with $x$ representing the laser direction, $y$ perpendicular to the burner surface and the origin at the center of the bluff body.

\subsection{Surface thermometry}

The TP coating used in this work was prepared by mixing a commercially available phosphorescence dye $\left(\mathrm{Mg}_{4} \mathrm{FGeO}_{6}\right.$ :Mn, Osram, SV067) with a water based binder (HPC binder, Zyp coatings), by a mass ratio of 1:10. A 4-mm-wide strip of this mixture was then spray-painted onto the surface of the base plate with an airbrush (Badger 100), as illustrated in Fig.1 1b. According to Ref. [23], no thermal gradient across the TP coating was observed with a layer thickness of $<20 \mu \mathrm{m}$. Therefore in this work, a controlled thickness of 10 to $15 \mu \mathrm{m}$ was achieved by repeated spray coating (each layer was dried with a heat gun) and was measured with an electromagnetic thickness gauge (Sauter TE $1250-0.1 F N)$. Afterwards, the coating was stabilized in an oven for 2 hours at $300{ }^{\circ}$. Since the bluff body could not be taken off the combustor for spray coating, the same TP mixture was brushed onto the tip of the bluff body and dried by a heat gun.

The strong temperature dependence of phosphorescence lifetime [24] was utilized in this work to obtain the surface temperature. In order to resolve the phosphorescence decay, the exposure of the CMOS camera was triggered repeatedly at $70 \mathrm{kHz}$ with a gate width of about $14 \mu \mathrm{s}$. The synchronization between laser excitation and camera gating, as shown in the timing diagram in Fig.3, was achieved by a delay generator acting as a master clock. The phosphorescence signal from each laser pulse was divided into 70 sequential frames. An example of phosphorescence signal from a single frame at room temperature is provided in Fig,1 $\mathrm{b}$, with a sketch of the burner surface as a reference (not to scale). A background image was obtained prior to each set of measurements by blocking the laser beam. By integrating across the width, the background-subtracted, approximately $80 \mathrm{~mm}$ by $1 \mathrm{~mm}$ phosphorescence signal was converted into a 1-D profile. A software binning of 10 pixels along the $x$-axis was also adopted to smooth the signal, resulting in a spatial resolution of $0.94 \mathrm{~mm}$.

Reference temperature in the range of $450 \mathrm{~K}$ to $850 \mathrm{~K}$ was obtained by performing the same measurement described above on the base plate placed inside a furnace with an optical access. Laser alignment was arranged to maintain the same laser path length post sheet optics (hence the 
(a)

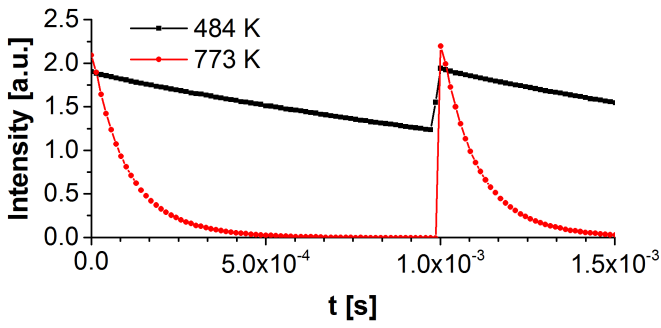

Figure 4:

Examples of data processing for phosphorescence signal obtained from the calibration furnace at two different temperatures

(a) Time traces of phosphorescence signal at the calibration point

(b) Single-exponential fit to phosphorescence decay traces

(c) Histograms of fitted decay time obtained from 250 successive laser shots

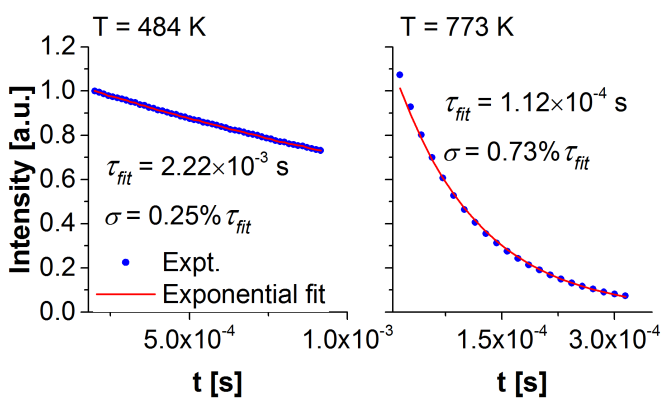

(c)

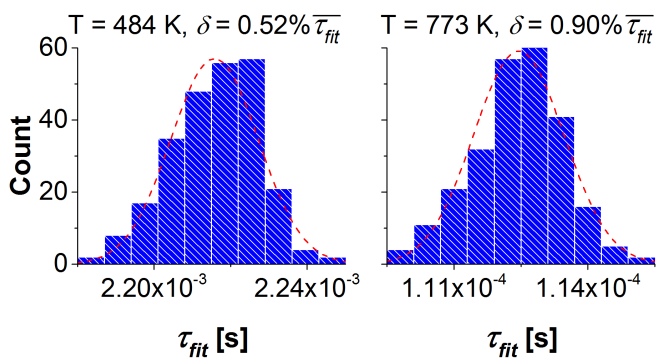

laser sheet thickness) for both the measurements on the combustor surface and the calibration in the furnace. In addition, a standalone thermocouple was installed to monitor temperature at the TP coating, minimizing the influence of potential temperature gradient inside the furnace.

\section{Calibration}

For calibration, the furnace was first heated to $850 \mathrm{~K}$, data were then acquired with 5-50 K intervals during the slow cooling ramp. At each reference temperature (from thermocouple readout), 250 successive laser shots were fired and a total of 17500 frames were acquired. Each frame was then converted into a 1-D profile following the procedure described in Section 2.3. However, only the data from a single point (denoted as the "calibration point") closest $(\sim 1 \mathrm{~cm})$ to the thermocouple were used.

Figure 4 a plots time traces of phosphorescence signal from the first two laser pulses at the calibration point at two reference temperatures. It shows that at $\mathrm{T}=773 \mathrm{~K}$, phosphorescence decays to zero within the interval of the laser pulses $(1 \mathrm{~ms}$ at $1 \mathrm{kHz})$. As temperature decreases and phosphorescence lifetime increases, the signal does not decay completely, as in the case of $\mathrm{T}=484 \mathrm{~K}$, but rather becomes a background to the signal from the subsequent laser shots. If the decay of phosphorescence follows a mono-exponential function (a sufficient assumption for this particular phosphor based on our experience), one can express the phosphorescence signal $\left(S_{P}\right)$ at a given 


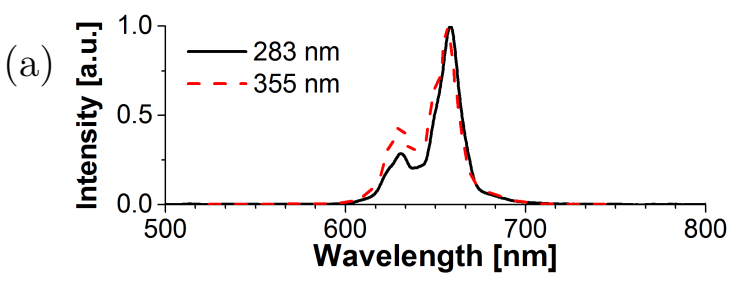

Figure 5:

(a) Comparison of phosphorescence spectra at room temperature from laser excitation at 283 $\mathrm{nm}$ and $355 \mathrm{~nm}$

(b) Measured phosphorescence decay as a function of recorded temperature in the calibration furnace

(c) Uncertainties in temperature inference

(d) Measurement sensitivity

(b)

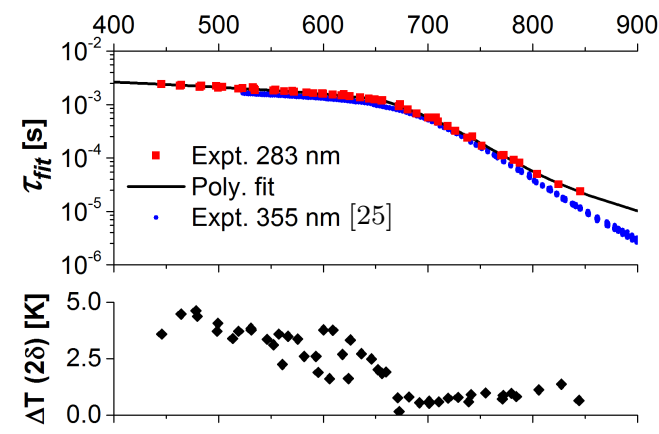

(d)

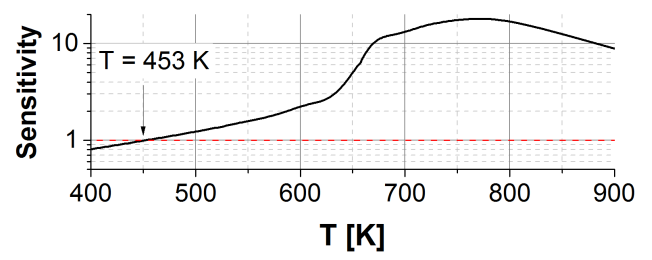

interval between two laser pulses as (omit the laser excitation process):

$$
S_{P}=\sum_{i=0}^{N} A_{i} \cdot \exp \left(-\frac{t+i \cdot \Delta t}{\tau_{i}}\right)
$$

The terms with $i>0$ represent the residual signal from previous pulses, with $\Delta t=1 \mathrm{~ms}$ here. At constant temperature conditions (i.e., $\tau_{i}$ remains unchanged during acquisition of one data set) as in the furnace, it is clear from Eq.(1) that measured decay time should not be affected by signal overlap. In this work, the decay time of phosphorescence was inferred from mono-exponential fit to the obtained signal. The fitting was conducted to each segment of the signal traces (i.e., 70 frames) corresponding to each laser shot. In addition, only the data points between $90 \%$ and $5 \%$ of the peak intensity were used for the fit.

Figure $4 \mathrm{~b}$ provides two fitting examples for the two temperature cases shown in Fig,4a. $\sigma$ represents the 1-standard deviation error on the fitted $\tau_{f i t}$. As can be seen, good fits were achieved for both cases, independent of the amount of signal overlap. The results also justify the use of a mono-exponential fit. The uncertainty of the calibration was estimated based on the standard deviation $(\delta)$ of 250 repetitive measurements, as shown in Fig, 4. As can be seen, the fluctuations in repeatedly measured phosphorescence decay are close to the uncertainties of the decay fit, see Fig.4 $\mathrm{b}$. This suggests that at stable conditions inside a furnace, the statistical error of this measurement approach is negligibly small.

The calibration results are summarized in Fig, 5 and compared with those obtained in Ref. [25] for the same TP but with a $355 \mathrm{~nm}$ excitation and point-wise signal collection based on a photomultiplier tube (PMT). Figure 5a plots normalized emission spectra from the TP coating at room temperature subjected to two different excitation wavelengths, both were taken with the same Avantes spectrometer (AvaSpec 3648). Minor difference can be observed regarding the ratio of the 
two main emission branches. All measurement results from the furnace at various reference temperatures are included as red square symbols in Figure 5b, which appear in good agreement with data obtained with $355 \mathrm{~nm}$ excitation (blue dots) except at T>800 K. Similar discrepancy was also observed in Ref.[11] and was attributed to the differences in the transfer function of the PMT and the highspeed camera. A continuous calibration curve (solid black line) was then obtained by fitting the current results to a polynomial function. The calibration uncertainties at various reference temperatures were estimated as described in Fig.4 c. 2-standard deviation of the repeatedly measured decay time ( $2 \sigma$, equivalent to a $95 \%$ confidence interval) were converted into uncertainty in temperature $( \pm \Delta \mathrm{T})$ using the calibration curve, and plotted in Fig.5c (only the positive part is plotted as the negative part has nearly identical value). As can be seen, $\Delta \mathrm{T}$ remains small throughout the full range of reference temperatures and reaches its minimum at around $680 \mathrm{~K}$.

To access quantitatively the temperature sensitivity $(k)$ of the current $\mathrm{TP}$, we define here:

$$
k \equiv \frac{|d \tau / \tau|}{|d \mathrm{~T} / \mathrm{T}|},
$$

which is essentially the normalized derivative of $\tau$ with regard to T. A higher $k$ value indicates higher temperature sensitivity, i.e., smaller variation in temperature can be accurately measured. Based on the calibration curve from Fig $4 \mathrm{~b}$ (the polynomial fit), $k$ was computed and is plotted in Fig 4 d. As can be seen, the current TP reaches its peak sensitivity at 700-800 K, consistent with trend in $\Delta \mathrm{T}$ shown in Fig 4 c. At below about $450 \mathrm{~K}, k$ becomes less than one and the technique is therefore considered inaccurate.

It should be pointed out that, although the signal overlap can be avoided by choosing a much slower laser repetition rate, the final choice of $1 \mathrm{kHz}$ was a compromise considering:

1) the desired time resolution for resolving the details of flame transitions

2) the minimum time resolution for resolving the potential influence of $\mathrm{PVC}$ (with $\mathrm{f}=490 \mathrm{~Hz}$ [4]) at the operating condition

3) the laser energy, which decreases substantially with reducing repetition rate for this particular system

4) the extent of signal overlap, as a result higher laser frequency was not used

As for the last point, excessive overlap would restrict the temporal range of data points available for an accurate decay fit. In the temperature range from 500 to $800 \mathrm{~K}$ (estimated surface temperature), phosphorescence does not last longer than a few milliseconds. Temperature is not expected to undergo a significant change over this period even on the combustor surface exposed to turbulent flames. Therefore the above assumption of a constant $\tau$ in Eq.(1) also holds well for measurements conducted in the combustor.

\section{Results}

Figure 6 presents two samples of results from single-shot, simultaneous OH PLIF and 1-D surface thermometry in the combustor at the operating condition detailed in Section 2.1. Both were chosen from one set of measurements of 8100 continuous laser shots. 1-D profiles of phosphorescence decay time were derived from more than half of a million frames following the procedures described in Section 2.3 and Fig. 4. The decay time was then converted to temperature using the calibration curve obtained in Fig. $4 \mathrm{~b}$ (the polynomial fit). The OH PLIF images went through a post processing routine including (1) background (dark current) subtraction, (2) laser profile correction (as described in Section 2.2 and (3) a 2 by 2 software binning to improve signal to noise. In the OH PLIF images, 
Figure 6:

Representative results of instantaneous $\mathrm{OH}$ signal distribution and surface temperature during
(a) V-flame and
(b) M-flame

(a)

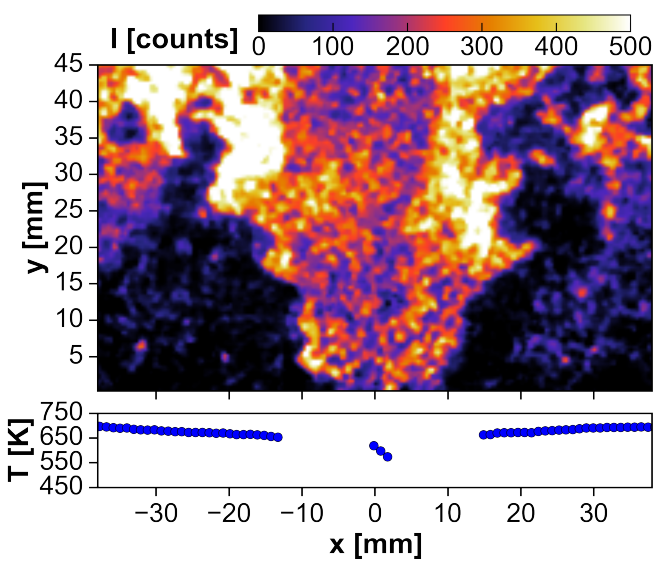

(b)

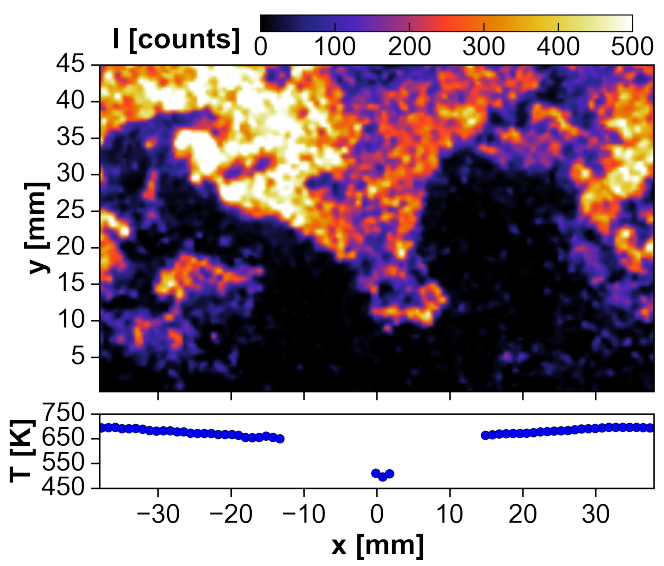

regions without any signal represent mostly unburned gas mixtures with $\mathrm{T}<1500 \mathrm{~K}$. High OH signal can be found mostly along the junctions of unburned and burned gas (with low-to-medium signal level) and is considered a good marker of reaction zones in this type of flames [4, 26. As can be seen, at the incidence when flame is attached at the bluff body (V-flame, Fig.6a), temperature at the surface of the bluff body is much higher, by almost $100 \mathrm{~K}$, than when flame is lifted (Mflame, Fig.6b). On the other hand, temperature profiles on the base plate of the combustor appear symmetric and nearly indistinguishable between the two flame shapes.

Figure $7 \mathrm{a} \& \mathrm{~b}$ plots time traces of spatially integrated $\mathrm{OH}$ signal and surface temperature at $\mathrm{x}=0 \mathrm{~mm}$ (the tip of the bluff body), depicting multiple events of flame transitions between $\mathrm{V}$ - and M-shapes. The results come from the same data set as Fig,6. Note that surface temperature was derived from each single shot measurement and the results in Fig $7 \mathrm{~b}$ were not further smoothed in time. The integration window for $\mathrm{OH}$ signal was $8-\mathrm{mm}$ wide by $5-\mathrm{mm}$ tall right above the tip of the bluff body, and is shown schematically as the white rectangle in Fig 6 a. While near zero $\mathrm{OH}$ signal indicates a lifted M-flame, sustained non-zero $\mathrm{OH}$ signal in the integration window points to an attached V-flame. From Fig.7b, it appears that the temperature at the tip of the bluff body experiences rather volatile changes (peak to peak variation at about $100 \mathrm{~K}$ ) in this bi-stable flame. The transitions between two flames are rather rapid, with a maximum temperature drop of about $50 \mathrm{~K}$ within $50 \mathrm{~ms}$ as the flame switches from $\mathrm{V}$ - to M-shape at around $\mathrm{t}=0.6 \mathrm{~s}$. During the V-flame period (none-zero $\mathrm{OH}$ signal), surface temperature at the tip of the bluff body slowly increases as if trying to reach a steady state. The opposite happens when flame switches to M-shape (near-zero $\mathrm{OH}$ signal). An incomplete flame transition event at about $\mathrm{t}=3.1 \mathrm{~s}$ (sudden spike in $\mathrm{OH}$ signal) was also captured in measured temperature. Notice that surface temperature at this instance is higher 
Figure 7:

Temporal traces at $\mathrm{x}=0 \mathrm{~mm}$

(a) Integrated $\mathrm{OH}$ signal

(b) Surface temperature during flame transitions

(c) Temperature uncertainty from decay fit

(d) Temperature fluctuation

(b)

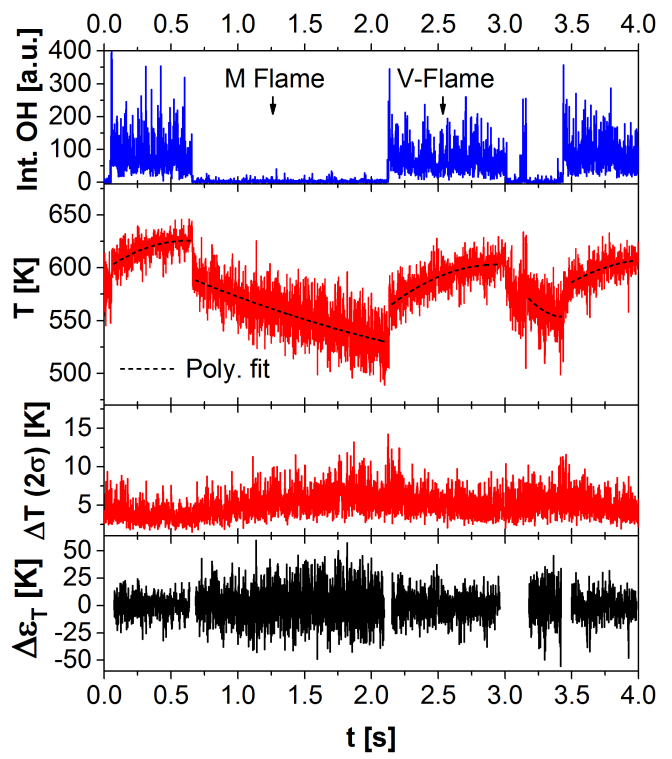

Figure 8:

FFT of the temperature at $\mathrm{x}=0 \mathrm{~mm}$ during different flame shapes

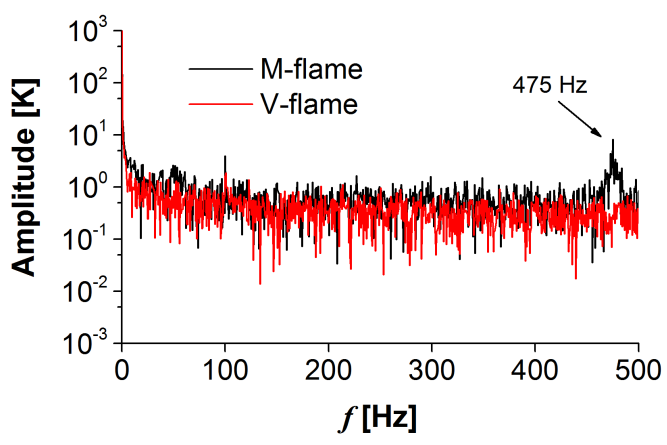

than the two complete $\mathrm{M}$ to $\mathrm{V}$ transitions at $\mathrm{t}=2.1 \mathrm{~s}$ and $\mathrm{t}=3.4 \mathrm{~s}$, suggesting that there might exist a favorable range of temperature for flame transitions to occur.

Figure $7 \mathrm{c}$ plots the uncertainties in measured temperature estimated based on the $95 \%$ confidence interval of the decay fit $(2-\sigma)$ as described in Fig. $4 \mathrm{~b}$. Their rather small magnitudes $(<15 \mathrm{~K})$ do not seem to completely account for the rather large fluctuations seen in the measured temperature in Fig.7b, especially when the flame is lifted. In order to obtain a quantitative measure of the fluctuation, sections of the temperature time trace were fitted to polynomial functions, which are plotted as dashed lines in Fig.7p. The residuals of the fit (i.e., T-T ${ }_{f i t}$ ), $\Delta \epsilon_{\mathrm{T}}$, are shown in Fig.7 $\mathrm{d}$. Surface temperature during the V-flame period appears much more stable than during M-flame. As mentioned in Section 1, when flame transitions from V-to M-shape, a PVC also forms and exerts a strong influence on combustion dynamics. Further more, it has been shown that PVC originates right at the exit of the nozzle [4, suggesting that it can have direct impact on surface temperature on the bluff body as well. The observed larger fluctuation in surface temperature in Fig.7f could therefore be related to the existence of PVC.

Figure 8 plots the FFTs of the time-resolved temperature shown in Fig.7b. FFTs were computed for both $\mathrm{V}$ - and M-flames by only including the sections containing either flame shape. A peak at about $475 \mathrm{~Hz}$ is only visible during the M-flame period, which is in good agreement with the PVC frequency of $490 \mathrm{~Hz}$ measured at the same condition [4]. The small discrepancy could come from the relatively low laser repetition rate $(1 \mathrm{kHz})$ adopted in this work, which is marginal to accurately resolve the peak. Nonetheless, the result demonstrates the potential of the diagnostic apparatus developed in this work in capturing small and fast surface temperature variations.

On the other hand, on the base plate of the combustor, temperature has a much milder response 
Figure 9:

Temporal traces at $\mathrm{x}=27 \mathrm{~mm}$

(a) Integrated $\mathrm{OH}$ signal

(b) Surface temperature during flame transitions

(c) Temperature uncertainty from decay fit

(d) Temperature fluctuation

(b)

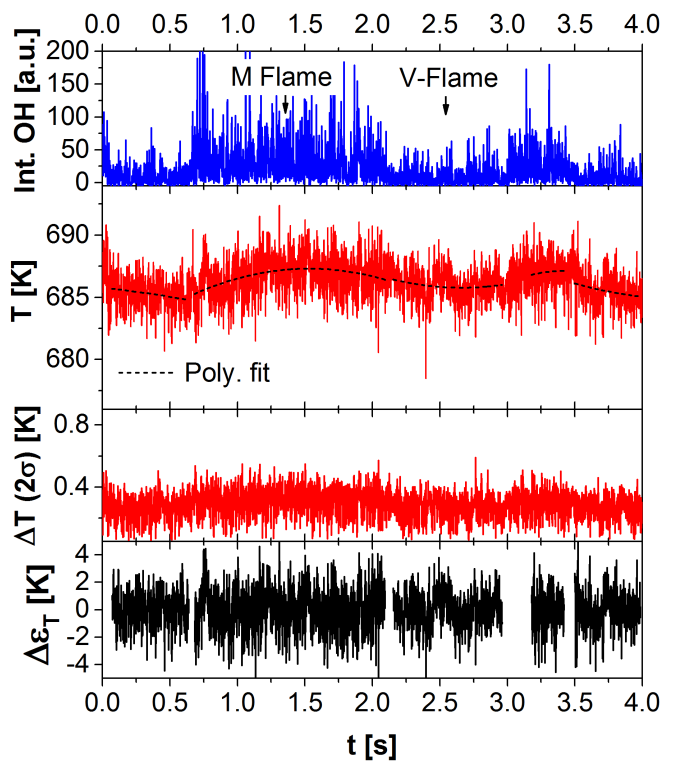

to flame transitions and remains in general quite stable. This is shown in Fig. 9 $\mathrm{b}$, which plots the time-evolution of surface temperature at $\mathrm{x}=27 \mathrm{~mm}$ on the base plate. As the indicator of flame transitions, integrated $\mathrm{OH}$ signal is plotted in Fig. 9a with the same size integration window as in Fig. $7 \mathrm{a}$ but centered at $\mathrm{x}=27 \mathrm{~mm}$. As expected, $\mathrm{OH}$ signal behaves in the opposite way compared to that above the bluff body, with a sustained increase in intensity indicating an M-flame, due to more hot gas in contact with the burner base plate. However, such variation in $\mathrm{OH}$ signal is much less pronounced compared with Fig. $7 \mathrm{a}$, suggesting a more stable combustion environment at this location. This is echoed in measured surface temperature: although temperature can be discerned to increase/decrease with flame transitions, the variation is within just $10 \mathrm{~K}$. In the same manner described in Fig. 7, the estimated measurement errors and temperature fluctuations are included in Fig. 9 $\& d$, both of which are significantly smaller compared to the results at $\mathrm{x}=0 \mathrm{~mm}$. In addition, no peak such as in Fig. 8 was detected in the spectral analysis of temperature measured on the base plate of the combustor.

In summery, temperature on the tip of the bluff body appears to be highly sensitive to flame transitions. This is likely due to the fact that the bluff body is constantly cooled by the cold unburned gas exiting the nozzle. At the same time, it experiences dynamic changes from flame attachment/detachment and the formation/suppression of PVC. On the other hand, the base plate might have a higher inertia to temperature variations since it is in contact with the bulk body of combustor, which is not actively cooled.

\section{Discussion}

In this section, the possible influence of measurement uncertainty is further examined. The focus is placed on the results from the tip of the bluff body, where larger temperature fluctuations during the M-flame period might also be caused by the lower sensitivity of the TP at low temperature ranges as shown in Fig. 5 d.

Figure 10 shows a scatter plot of the temperature fluctuations, $\Delta \epsilon_{\mathrm{T}}$ in Fig.7d, versus measured temperature in Fig.7b. The data were sorted into two groups depending on the flame shape, blue hollow symbols represent the V-flame and red solid symbols represent the M-flame. Each side of the scatter plot shows the histogram of all the data belonging to the same flame shape. It is clear that surface temperature on the tip of the bluff body during V-flame appears more stable, 
Figure 10: Comparison of temperature fluctuation during $\mathrm{V}$ - and M-flames

Figure 11:

Comparison of temperature fluctuation and sensitivity in the same temperature range
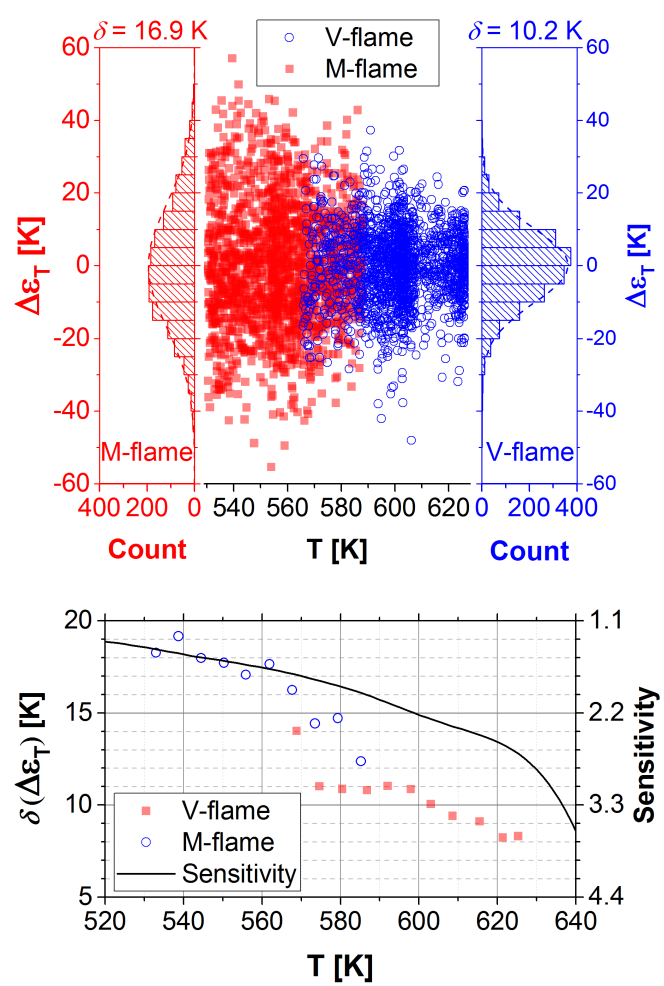

with a standard deviation $(\delta)$ of $10.0 \mathrm{~K}$ compared with $16.9 \mathrm{~K}$ for the M-flame. However, from the overlap region of the two groups of data at around $580 \mathrm{~K}$ (therefore same measurement sensitivity), temperature measured during the V-flame has smaller scatter compared with those from the Mflame. This indicates that the large temperature fluctuation in the M-flame is not completely due to the sensitivity of the TP. But it is at least partially reflecting the more dynamic environment when flame is lifted, as the cooling effect from the unburned gas exiting the nozzle dominates over the heating effect of recirculated burned gas. This is evident from the fast decreasing of surface temperature on the tip of the bluff body in Fig.7p. Whereas during V-flame, the tip of the bluff body may be in constant contact with the hot flame, which provides a stable local heating source. Also note that these fluctuations are in general much larger than those measured in the stable furnace at the same temperature range $(<5 \mathrm{~K}$, refer to Fig.5 $)$, another indication that the turbulent environment in the combustor can in general contribute to the larger fluctuation in measured temperature.

A more quantitative perspective of the observations in Fig,10 is provided in Fig,11, which compares the temperature fluctuation with the sensitivity calculated in Fig. 5 d. Standard deviations of the temperature fluctuations (representing the degree of temperature fluctuation) were calculated by sorting $\Delta \epsilon_{\mathrm{T}}$ shown in Fig.10 into temperature groups stepped by $5 \mathrm{~K}$. A trend of decreasing measured fluctuation with increasing temperature can be clearly observed. Since the fitting uncertainties in measured $\tau$ are small and can be assumed as constant, according to Eq. (2), $\delta\left(\Delta \epsilon_{\mathrm{T}}\right)$ should then scale inverse proportionally with sensitivity if the measured fluctuation is controlled solely by the sensitivity of the TP. For order of magnitude comparison, the scale of the sensitivity (the axis on the right) was adjusted to match that of $\delta\left(\Delta \epsilon_{\mathrm{T}}\right)$ (the axis on the left). In the same temperature range shown here, sensitivity of the TP does not increase by the same factor of reduction seen in measured temperature fluctuation. Therefore, the surface temperature is indeed more stable during V-flame period. Additionally, there appears to be a hysteresis in the temperature range where the two flames overlap, a phenomenon that cannot be attributed to measurement accuracy (due to 
same temperature sensitivity) but is yet another evidence that M-flame constitutes a more dynamic environment, likely caused by PVC as discussed in Section 4.

Nonetheless, the above analysis suggests that the measurement accuracy can be improved by choosing a TP that is more sensitive in the temperature range of 500-600 K. Furthermore, by choosing a TP that has a shorter decay time in this temperature range, a higher laser repetition rate could also be implemented to better capture the effect from PVC.

\section{Conclusions}

Simultaneous 1-D surface thermometry and OH planar laser-induced fluorescence (OH PLIF) based on a single UV laser source was performed at $1 \mathrm{kHz}$ in a swirl-stabilized gas turbine model combustor. The surface thermometry utilizes a commercially available thermographic phosphor (TP) and derives temperature from the decay of the phosphorescence recorded by a CMOS camera operated at $70 \mathrm{kHz}$. At the chosen operating condition, the flame is bi-stable, exhibiting intermittent transitions between $\mathrm{V}$ - and M-shapes. The response of surface temperature on the base plate and the bluff body of the combustor to such transient events are captured in a long recording of over half of a million frames (approximately $8 \mathrm{~s}$ ) and is correlated to the flame shapes monitored by $\mathrm{OH}$ PLIF.

The results demonstrate that, on the tip of the bluff body (1) temperature can vary by as much as $100 \mathrm{~K}$ between $\mathrm{V}$ - and M-flames, (2) sudden temperature increase/decrease up to $50 \mathrm{~K}$ within $50 \mathrm{~ms}$ can occur during the transition between two flame shapes, (3) temperature measured during the M-flame period exhibits a peak at $475 \mathrm{~Hz}$ in the frequency domain, in good agreement with previously measured frequency of the precessing vortex core (PVC) at the same operating condition. This peak does not exist for temperature measured during the V-flame period, since PVC is suppressed when flame transitions from M- to V-shape. While at the same time on the base plate of the combustor, only minor variations in temperature is observed and the PVC peak is completely missing.

The uncertainty of the measurements conducted in the combustor are examined from various perspectives. The analysis shows that large fluctuation in measured temperature is not completely due to the sensitivity of the technique, but also reflects at least partially the volatile environment in the turbulent flame.

Together with previous highspeed measurements of velocity and scalar fields, the current results are being analyzed in an ongoing effort to achieve a comprehensive understanding of the transitions in flame shapes at the bi-stable operating condition.

\section{Acknowledgment}

The authors would like to thank Michael Stöhr for his technical support. Zhiyao Yin acknowledges the financial support within the Helmholtz Postdoc Programme (Grant PD-112).

\section{References}

[1] A. Ketelheun, G. Kuenne, J. Janicka. Heat transfer modeling in the context of large eddy simulation of premixed combustion with tabulated chemistry. Flow Turbul Combust 91 (2013) 867-893.

[2] Z. Duan, B. Shaffer, V. McDonell, G. Baumgartner, T. Sattelmayer. Influence of burner material, tip temperature, and geometrical flame configuration on flashback propensity of h2air jet flames. J. Eng. Gas Turbines Power 136 (2014) 021502. 
[3] F. Proch, A. Kempf. Modeling heat loss effects in the large eddy simulation of a model gas turbine combustor with premixed flamelet generated manifolds. Proc. Combust. Inst. 35 (2015) $3337-3345$.

[4] K. Oberleithner, M. Stöhr, S. H. Im, C. M. Arndt, A. M. Steinberg. Formation and flameinduced suppression of the precessing vortex core in a swirl combustor: Experiments and linear stability analysis. Combust. Flame 162 (2015) 3100 - 3114.

[5] Q. An, W. Y. Kwong, B. D. Geraedts, A. M. Steinberg. Coupled dynamics of lift-off and precessing vortex core formation in swirl flames. Combust. Flame 168 (2016) 228 - 239.

[6] N. Syred. A review of oscillation mechanisms and the role of the precessing vortex core (pvc) in swirl combustion systems. Prog. Energy Combust. Sci. 32 (2006) 93 - 161.

[7] M. Stöhr, I. Boxx, C. D. Carter, W. Meier. Experimental study of vortex-flame interaction in a gas turbine model combustor. Combust. Flame 159 (2012) 2636 - 2649. Special Issue on Turbulent Combustion.

[8] M. Aldén, A. Omrane, M. Richter, G. Särner. Thermographic phosphors for thermometry: A survey of combustion applications. Prog. Energy Combust. Sci. 37 (2011) 422 - 461.

[9] J. Brübach, C. Pflitsch, A. Dreizler, B. Atakan. On surface temperature measurements with thermographic phosphors: A review. Prog. Energy Combust. Sci. 39 (2013) 37 - 60.

[10] A. H. Khalid, K. Kontis. 2d surface thermal imaging using rise-time analysis from laser-induced luminescence phosphor thermometry. Meas. Sci. Technol. 20 (2009) 025305.

[11] T. Kissel, E. Baum, A. Dreizler, J. Brübach. Two-dimensional thermographic phosphor thermometry using a cmos high speed camera system. Appl. Phys. B 96 (2009) 731-734.

[12] S. Someya, M. Uchida, K. Tominaga, H. Terunuma, Y. Li, K. Okamoto. Lifetime-based phosphor thermometry of an optical engine using a high-speed CMOS camera. Int. J. Heat Mass Transfer 54 (2011) $3927-3932$.

[13] S. Someya, H. Furutani, K. Okamoto. Instantaneous phosphor thermometry applicable to walls exposed to flames. Exp. Therm Fluid Sci. 47 (2013) $224-231$.

[14] S. Someya, Y. Okura, T. Munakata, K. Okamoto. Instantaneous 2d imaging of temperature in an engine cylinder with flame combustion. Int. J. Heat Mass Transfer 62 (2013) 382 - 390.

[15] M. Euler, R. Zhou, S. Hochgreb, A. Dreizler. Temperature measurements of the bluff body surface of a swirl burner using phosphor thermometry. Combust. Flame 161 (2014) 2842 2848.

[16] N. Fuhrmann, E. Baum, J. Brübach, A. Dreizler. High-speed phosphor thermometry. Rev. Sci. Instrum. 82 (2011) 104903.

[17] W. Meier, P. Weigand, X. Duan, R. Giezendanner-Thoben. Detailed characterization of the dynamics of thermoacoustic pulsations in a lean premixed swirl flame. Combust. Flame 150 (2007) $2-26$. 
[18] A. Steinberg, C. Arndt, W. Meier. Parametric study of vortex structures and their dynamics in swirl-stabilized combustion. Proc. Combust. Inst. 34 (2013) 3117 - 3125.

[19] C. Dem, M. Stöhr, C. M. Arndt, A. M. Steinberg, W. Meier. Experimental study of turbulencechemistry interactions in perfectly and partially premixed confined swirl flames. Zeitschrift für Physikalische Chemie 229 (2015) 569-595.

[20] S. Roux, G. Lartigue, T. Poinsot, U. Meier, C. Bérat. Studies of mean and unsteady flow in a swirled combustor using experiments, acoustic analysis, and large eddy simulations. Combust. Flame 141 (2005) $40-54$.

[21] B. Franzelli, E. Riber, L. Y. Gicquel, T. Poinsot. Large eddy simulation of combustion instabilities in a lean partially premixed swirled flame. Combust. Flame 159 (2012) 621 - 637.

[22] J.-M. Lourier, C. Eberle, B. Noll, M. Aigner. Influence of turbulence-chemistry interaction modeling on the structure and the stability of a swirl-stabilized flame. in: ASME Turbo Expo 2015: Turbine Technical Conference and Exposition, American Society of Mechanical Engineers, pp. V04BT04A008-V04BT04A008.

[23] C. Knappe, M. Algotsson, P. Andersson, M. Richter, M. Tunér, B. Johansson, M. Aldén. Thickness dependent variations in surface phosphor thermometry during transient combustion in an HCCI engine. Combust. Flame 160 (2013) 1466 - 1475.

[24] N. Fuhrmann, J. Brübach, A. Dreizler. Phosphor thermometry: A comparison of the luminescence lifetime and the intensity ratio approach. Proc. Combust. Inst. 34 (2013) 3611 3618.

[25] Z. Yin, P. Nau, I. Boxx, W. Meier. Characterization of a single-nozzle FLOX ${ }^{\circledR}$ model combustor using khz laser diagnostics. in: Proc. ASME Turbo Expo 2015, Power for Land, Sea and Air, June 15-19, Montreal, Canada.

[26] R. Sadanandan, M. Stöhr, W. Meier. Simultaneous OH-PLIF and PIV measurements in a gas turbine model combustor. Appl. Phys. B 90 (2008) 609-618. 